Supporting Information

\title{
Energy-Loss Near-Edge Structures and Low-Loss Structures of Solid Electrolyte Interface Formed from Fluoroethylene Carbonate on Si Anode Clarified by DFT Calculations
}

Yuki Kamikawa, ${ }^{*},+, ;, *$ Koji Amezawa, and Kenjiro Terada ${ }^{\S}$

\#Institute of Multidisciplinary Research for Advanced Materials, Tohoku University, 2-1-1

Katahira, Aoba-ku, Sendai 980-8577, Japan

§International Research Institute of Disaster Science, Tohoku University, 468-1-S403, Aoba, Aramaki, Aoba-ku, Sendai 980-8572, Japan

\#Nissan Motor Co., Ltd. 1-1-1, Takashima, Nishi-ku, Yokohama 220-8686, Japan

${ }^{\dagger}$ Research Center, Envision AESC Japan Ltd., 2-10-1 Hironodai, Zama 252-0012, Japan 


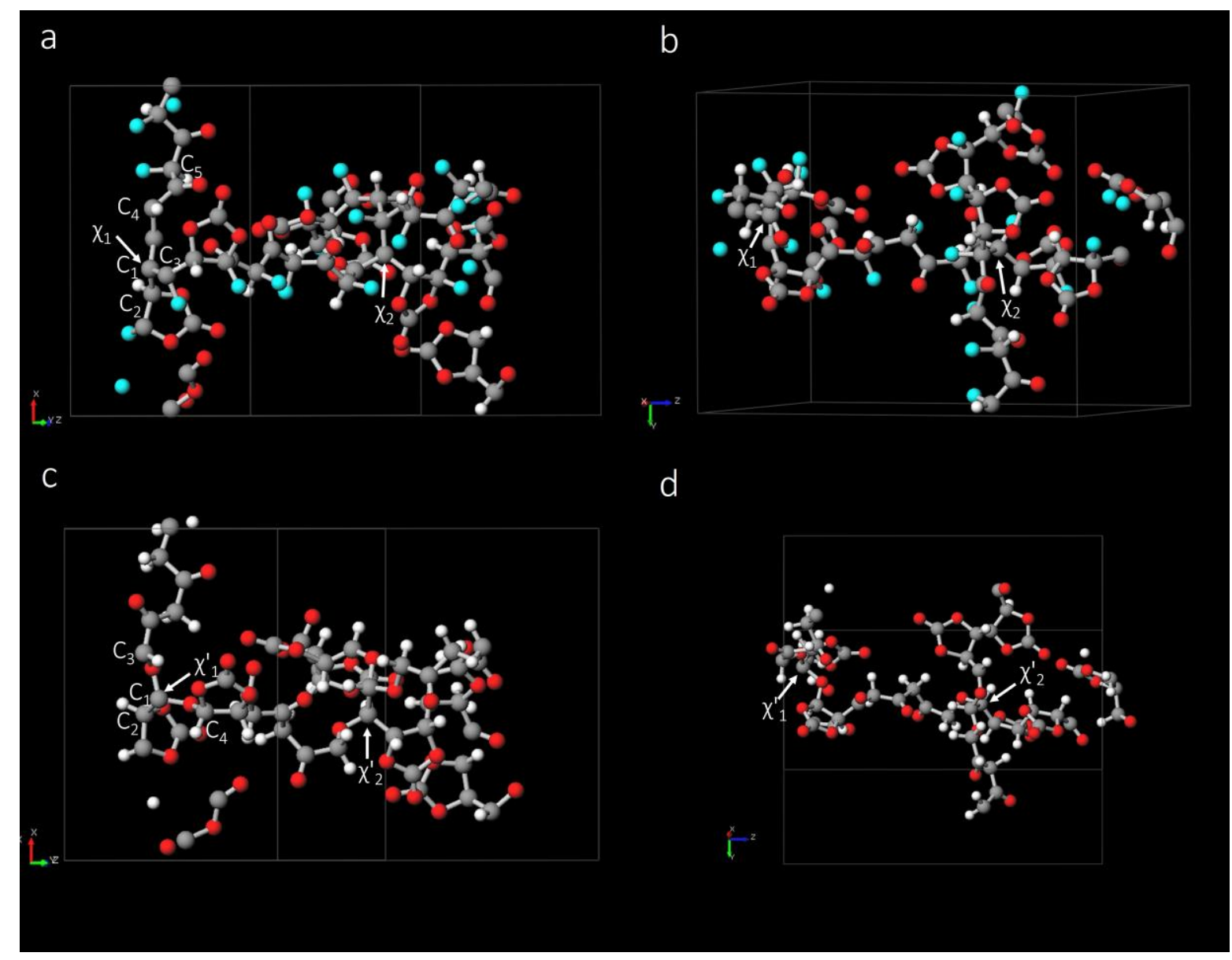

Figure S1. Atomic structures of (a and b) crosslinked polymerized fluoroethylene carbonate [crosslinked poly(FEC)] branched at $\chi_{1}$ and $\chi_{2}$, and (c and d) poly(vinylene carbonate) $[$ poly $(\mathrm{VC})]-(\mathrm{CHCHO})_{n}$ branched at a $\mathrm{C}(\mathrm{OR})_{2}$ binding site $\left(\chi_{1}^{\prime}, \chi_{2}^{\prime}\right)$, with different rotations for each polymer. (a) and (c) also show the locations of the carbon atoms $\left(\mathrm{C}_{1}-\mathrm{C}_{5}[\mathrm{a}], \mathrm{C}_{1}-\mathrm{C}_{4}\right.$ [c]) at which the core-hole was constructed for core-loss calculations. The gray, red, white, and blue colors represent $\mathrm{C}, \mathrm{O}, \mathrm{H}$, and $\mathrm{F}$ atoms, respectively. 